Brève analyse des liens cultures - environnements développements dans une perspective d'éducation relative à l'environnement

Carmen Ramos et Christian Souchon

\author{
(2) OpenEdition \\ Journals \\ Édition électronique \\ URL : http://journals.openedition.org/ere/5446 \\ DOI : $10.4000 /$ ere. 5446 \\ ISSN : 2561-2271 \\ Éditeur \\ Centr'ERE
}

Référence électronique

Carmen Ramos et Christian Souchon, «Brève analyse des liens cultures - environnements développements dans une perspective d'éducation relative à l'environnement », Éducation relative à l'environnement [En ligne], Volume 4 | 2003, mis en ligne le 14 septembre 2003, consulté le 18 juin 2020. URL : http://journals.openedition.org/ere/5446 ; DOI : https://doi.org/10.4000/ere.5446 


\title{
Brève analyse des liens cultures - environnements - développements dans une perspective d'éducation relative à l'environnement
}

\author{
Carmen Ramos et Christian Souchon
}

1 Dans la naissance et la formalisation de l'éducation relative à l'environnement (ERE), il est facile de déceler bien d'autres « racines » que celles liées à une démarche éducative. Un courant essentiel est certainement celui qui renvoie aux relations Homme-Nature.

2 En France par exemple, l'ouvrage de Jean Dorst (1965) "Avant que nature meure ", repris et résumé dans une version intitulée «La nature dé-naturée : pour une écologie politique », garde encore aujourd'hui son acuité d'analyse et sa valeur de cri d'alarme : on notera au passage la force du sous-titre "pour une écologie politique» et les implications qu'il comporte.

3 L'inquiétude vis-à-vis des atteintes à la nature, à la faune et à la flore et l'idée de protection s'était déjà exprimée bien avant en France par l'établissement dans la Forêt de Fontainebleau de réserves nommées alors «artistiques » à cause de l'influence des peintres de Barbizon. Par ailleurs, Jean-Henri Fabre, célèbre naturaliste de Provence (1823- 1915), manifestait déjà en 1899 une inquiétude plus générale en critiquant dès cette époque, une certaine vision du progrès : «le progrès amène de nouveaux besoins coûteux [...] une nation de progrès en progrès, s'achemine au suicide » (Fabre, 1989, p. 18 ; réédition de l'œuvre originalement parue en 1899).

4 Dans les années 1960 aux États-Unis s'est créé un mouvement dit consumériste porteur d'une réflexion sur ce citoyen-consommateur, qui aujourd'hui se manifeste surtout par ses inquiétudes égoïstes vis-à-vis de son confort et de sa santé plutôt que par de véritables préoccupations écologiques. C'est aussi à partir des années 1960 que se manifeste un autre courant plus large, allant au-delà du seul cercle des naturalistes, en constituant une véritable prise de conscience écologique (Deléage et Grinevald, 1993). Une des composantes de ce courant met l'accent sur le risque d'épuisement des 
ressources non renouvelables; le fameux rapport « Halte à la croissance » (Meadows et Delaunay, 1972), certes mal étayé et fragile dans ses méthodes de prévision (d'où les multiples critiques dont il a été l'objet), porte sur ce point essentiel. Des inquiétudes du même type sont aussi exprimées face à une démographie à croissance exponentielle, notamment par la parution des « 3P » (Population, plenty and poverty) de Erlich et Erlich (1998), même si un agronome comme Klatzmann (1975) affirme que l'on pourra facilement « nourrir dix milliards d'hommes».

5 Est-il possible de retrouver dans ces manifestations de prise de conscience des problèmes environnementaux et de gestion des ressources, les fondements culturels de l'ERE? Quelle influence pouvons-nous y déceler pour analyser nos conceptions de l'ERE, les modeler au besoin et les traduire dans une pratique concrète?

\section{Comment nos références culturelles se traduisent- elles dans le concret de notre vie?}

6 À quelle culture appartenons-nous? Que représente pour nous, dans notre société moderne, industrielle et occidentale, «la culture »? L'usage le plus simple du mot est celui qui vaut, par exemple en France, son nom au Ministère de la Culture, lequel s'occupe des arts, des lettres, du patrimoine et aussi plus récemment de culture scientifique et technique. Ceci nous renvoie donc à l'admiration des produits de l'intelligence, des savoirs de plus en plus étendus et approfondis comme attributs essentiels de l'Homme cultivé.

7 Pour toucher à la vie quotidienne qui, sans que cela soit dit, fait référence à la culture influençant la société, on est obligé de se pencher sur le mot civilisation. Le «Larousse du XXe siècle» propose, après le sens agricole du mot culture, un sens figuré : «la culture des beaux-arts, des sciences »; puis il renvoie à instruction, éducation, car « la culture de l'esprit ennoblit le cœur » (Voltaire); il signale aussi que le mot s'emploie quelquefois d'après l'allemand kultur dans le sens de «civilisation ». En consultant un dictionnaire anglais, nous sommes également conduits aux mœurs, coutumes et à la civilisation : « les coutumes et la civilisation d'un temps ou de personnes donnés » (The Oxford Reference Dictionnary, 1986).

8 De quoi est donc faite notre culture et comment se manifeste-t-elle dans la vie de nos sociétés, à la fois parce qu'on s'y réfère ou bien parce que l'on en ignore délibérément certains aspects? D'où vient notre prétendue civilisation? Pour un Européen de l'Ouest, les références à un ancrage religieux judéo-chrétien, à un passé encore vivant de greco-latinité, à de brillantes époques d'activité intellectuelle (Renaissance, Siècle des Lumières), à l'émergence de systèmes sociétaux réfutant d'anciens modes de gouvernement (la Révolution, qui nous dote en France des trois fameux principes: liberté, égalité, fraternité) sont autant d'indices possibles des éléments de notre culture ou tout au moins de ce que nous en disons.

On peut sûrement alors s'accorder pour considérer que les lignes de conduite qui nous sont proposées comme essentielles pour nos comportements restent d'une part, des impératifs religieux, moraux et idéologiques et d'autre part, les exigences du bon exercice de la démocratie. Mais comment cela se traduit- il dans la vie quotidienne des sociétés et des individus? Que vénérons-nous? Quelles sont nos "idoles», nos aspirations? 
10 Force est de constater que notre civilisation repose aujourd'hui sur deux "piliers » principaux, fortement liés: l'un médiatique, l'autre économique. Les médias font émerger de petits dieux vivants dans le sport, le spectacle, dans la sphère de la "réussite sociale» et ils privilégient par ailleurs le spectaculaire (y compris le catastrophique) et l'insolite, au détriment de l'analyse, de la réflexion et de toute autre référence notamment éthique. Dans le domaine économique, à l'échelle de l'individu, la manifestation de l'ego renvoie bien loin la solidarité, l'égalité ou l'équité aux dépens de l'exercice de sa liberté. Pour la société, il n'est question que de profits obtenus, encore mieux dans le cadre de la mondialisation et grâce au libéralisme! Max Weber (1905) a déjà largement glorifié des pratiques économiques qui sont non seulement admises, mais encore facilitées par une approche religieuse. Pourtant, le philosophe Michel Serres nous montre bien qu'une certaine économie conduit à une sorte de culture de guerre notamment vis-à-vis de l'environnement :

Que l'on passe de la guerre aux rapports économiques et rien de notable ne change dans le raisonnement. Quirinus, dieu de la production, ou Hermès, qui préside aux échanges, ne peuvent endiguer la violence plus efficacement parfois que Jupiter ou Mars et usent, pour ce faire, des mêmes procédés que ce dernier. Dieu unique en plusieurs personnes, Mars appelle guerre ce que les deux premiers nomment concurrence : poursuite des opérations militaires par d'autres moyens, exploitation, marchandises, argent ou information. Plus caché encore, le vrai conflit reparaît. Se reconduit le même schéma: par leur laideur et les ordures qu'accidentellement ils répandent, les usines chimiques, les grands élevages d'animaux, les centrales atomiques ou les pétroliers géants ramènent la violence objective globale sans autres armes que la puissance de leur taille, ni autre finalité que la recherche, commune et contractuelle, de la dominance sur les Hommes. (Serres, 1990, p. 33-34)

11 Ainsi, la culture occidentale, autoproclamée comme moderne et civilisée, se traduit concrètement par une domination effective et pratiquement exclusive des préoccupations économiques. C'est de cela dont découle alors les problèmes environnementaux, la mauvaise gestion et le gaspillage des ressources, voire certains conflits et même le risque d'autodestruction de notre propre espèce. Les liens entre d'une part, la culture s'exprimant dans nos civilisations, et d'autre part, l'économie et ses conséquences environnementales, sont évidents et ceci doit nous faire réfléchir sur la valeur de notre culture et les rapports de celle-ci avec d'autres cultures souvent moins dévastatrices. Claude Lévi-Strauss (1955) a déjà montré que des cultures dites primitives valent bien la nôtre et sûrement mieux par certains aspects. Le désenchantement de Lévi-Strauss à la fois face aux disparitions de ces civilisations fortement liées à leur environnement naturel et face à l'image de nos bonnes consciences de «civilisés» au service du développement économique, n'a pas aujourd'hui de raison d'être atténué. Bien au contraire, partant de l'économie, le mot développement et surtout l'appellation développement durable viennent aujourd'hui allonger la chaîne conceptuelle.

12 Aujourd'hui, notre civilisation essentiellement marchande nous propose encore de " gérer » notre planète à l'aide de la notion de développement durable. Mais la tenue du Sommet de Johannesburg à la fin août - début septembre 2002, dix ans après celui de Rio, montre bien la faiblesse des bilans et la communauté éducative en ERE doit réclamer le débat non seulement sur la notion de développement durable elle-même, mais encore bien plus sur l'usage qui en est fait. Lucie Sauvé (2002) rappelle les réticences au sein de la communauté éducative en ERE, vis-à-vis de cette notion de développement durable : 
Face aux vives critiques qui ont fusé de toutes parts (chez les philosophes, les sociologues, les éducateurs, les économistes eux-mêmes, etc.) à l'égard de la proposition du développement durable, la tendance actuelle est de redéfinir, chacun à sa façon, ce concept obligé qui s'est désormais imposé dans les différentes sphères de la société : on convient ainsi que le développement durable ne serait pas un but clairement défini, mais un chemin pour y parvenir ; à chacun de le tracer à sa convenance. Après l'argument du consensus, ce nouvel argument de la légitimité, voire nécessité, d'une appropriation différenciée apparaît efficace pour promouvoir l'idée (désormais perçue comme incontournable) d'un « développement durable ", salvateur de l'humanité.

13 Cette attitude critique, qui s'oppose essentiellement à la présentation actuelle de la notion de développement durable et à l'usage qui en est fait, est parfaitement justifiée. En effet, le rapport Brundtland (Commission mondiale sur l'environnement et le développement, 1988) met de l'avant deux conditions nécessaires pour la mise en œuvre des politiques de développement durable. Tout d'abord, on doit admettre la libéralisation du commerce et donc le libéralisme économique. Ensuite, le développement ne peut se réaliser qu'en présence d'une forte croissance. S'il est évident que pour les pays pauvres et pour les pays dits "émergents » une certaine croissance est indispensable, il en va tout autrement pour les pays riches, car on peut dire que presque mécaniquement la croissance économique accroît les pollutions, les gaspillages et les impacts négatifs sur l'environnement.

On doit rappeler un débat plus ancien qui avait déjà eu lieu après la Conférence de Stockholm de 1972 et avait conduit à un examen sérieux de la distinction entre croissance et développement. Parmi ceux qui souhaitent la mise en œuvre du développement durable en admettant les postulats du rapport Brundtland (Commission mondiale sur l'environnement et le développement, 1988), Christian Brodhag (2002), en tant que président de la Commission française de développement durable, évoque aussi l'idée de croissance durable :

Bien que la polémique se soit éteinte, la référence à la croissance reste au cœur de certaines propositions. Il n'est pas souhaitable d'utiliser ce terme qui ouvre à nouveau un conflit ancien sur la croissance, et le mot durable qui y est accolé lui donne la signification d'une croissance continue du produit national brut sans autre interrogation sur le contenu de cette croissance. En effet, il sous-entend une croissance économique durable, qui ne prendrait pas en compte la dimension sociale. Le traité de Maastricht fait référence à ce terme de croissance durable.

Cet auteur montre clairement que ce débat croissance-développement reste gênant pour certains et qu'en réalité la sphère économique fait usage de la notion de développement durable non pas pour modifier ses pratiques vis-à- vis de l'environnement et des sociétés, mais, sous prétexte d'un consensus, pour cautionner des pratiques économiques non contrôlées. Il nous semble que malgré tout, on ne peut en ERE ne pas intégrer une réflexion sur une éducation à, pour ou vers le développement durable, surtout si l'on considère que ce domaine éducatif est maintenant proposé comme partie intégrante d'une éducation à l'exercice de la citoyenneté (Ziaka et coll., 2001). Le problème est de savoir comment procéder.

\section{Conséquences au niveau des conceptions de l'ERE}

Il est évident que, comme toute forme d'éducation allant au-delà des enseignements dogmatiques, sectorisés et visant uniquement une acquisition de «savoirs» et de 
concepts spécialisés dans certains champs disciplinaires, l'ERE participe de façon très pertinente au développement de l'individu; son but fondamental est celui du rapport de l'individu et du groupe social à l'environnement (Sauvé et coll., 2001). Pour affirmer le rôle social de l'ERE dans les possibilités qu'elle offre de favoriser l'action vis-à-vis et au sein des sociétés, il convient de rappeler certaines spécificités telles que :

- Parmi les objectifs, la priorité est accordée à ceux de modifications d'attitudes et de comportements comparativement à ceux d'acquisition de connaissances (non négligeables cependant en vue du développement de compétences).

- Certaines valeurs sont privilégiées comme étant davantage liées à l'ERE, surtout celles de responsabilité, de solidarité et de tolérance ${ }^{1}$, ce qui définit déjà une véritable philosophie de ce domaine éducatif.

- Une «chaîne» est utilisée comme processus pédagogique face à des situations environnementales complexes : « décrire, analyser, comprendre, agir ${ }^{2}$ (on pourrait ajouter évaluer), ce qui non seulement constitue une sorte de guide méthodologique, mais invite en outre à la participation citoyenne concrète.

- Des outils méthodologiques adaptés sont utilisés (par exemple, l'approche systémique, l'analyse conceptuelle, la maîtrise de l'information sur l'environnement, les jeux de rôle), qui permettent d'affiner une méthodologie entre autres pour décrire les situations et des problèmes, ainsi que pour préciser les acteurs en jeu et leurs rôles.

17 Ceci débouche sur une conception de l'ERE susceptible d'en faire le «noyau dur » d'une éducation à l'exercice de la citoyenneté en assurant bien sûr les liens indispensables avec d'autres domaines éducatifs (par exemple, la consommation, la santé et le développement).

L'irruption de la notion de développement durable dans sa présentation actuelle n'est pas sans poser problème par rapport à l'ERE et il est normal d'avoir, dans une réaction critique, à la fois une attitude lucide et un souci d'examiner les propositions avec le plus d'objectivité possible. Nous suivons bien en cela Lucie Sauvé (2002) :

Il est pourtant possible d'envisager une éducation relative à l'environnement qui, tout en considérant la perspective du développement durable (comme un important phénomène socio-historique), ne s'y enferme pas. L'éducation relative à l'environnement ne peut se déployer que dans un espace de critique sociale, sans entrave ; la relation à l'environnement n'est pas a priori une affaire de compromis social, encore moins de consensus planétaire. L'ERE accompagne et soutient d'abord l'émergence et la mise en œuvre d'un projet d'amélioration de son propre rapport au monde.

On doit bien noter que l'usage qui est fait par les gouvernants, les décideurs et les groupes économiques de cette notion de développement durable est tout à fait scandaleux : l'extrême indigence des bilans de RIO plus 10 préparés pour le Sommet de Johannesburg et le catalogue de nouveaux engagements (destinés à ne pas être tenus) sont largement accusateurs pour qui veut entendre et voir. Devant une situation de ce type, quel peut être notre rôle éducatif dans la communauté de l'ERE?

Pour répondre en partie à cette question, au-delà d'un débat de fond qui existe déjà par ailleurs, nous voudrions ici nous baser sur un exemple concret susceptible de fournir des éléments précis de réponse. L'idée directrice de la recherche de Carmen Ramos (2003) est essentiellement de pouvoir définir des objectifs visant à maîtriser pleinement certains concepts précis, tout en considérant leur complexité. Cette recherche aborde le thème de la forêt, thème à de multiples facettes. Il est d'une grande richesse autant dans des processus pédagogiques classiques que dans une vue d'utilisation en ERE. En 
choisissant ce thème et en se limitant aux termes du débat introduits dans cet article, il est possible de proposer quelques points montrant la pertinence de certaines activités pour atteindre certains objectifs dans une conception de l'ERE orientée vers une éducation citoyenne. Ce travail a montré que :

- Les usages et rôles de la forêt sont les premiers points à présenter concrètement. Dans nos forêts tempérées et gérées d'Europe, la production de bois et les loisirs semblent dominer. Pour quelques peuples autochtones d'Amazonie, la forêt correspond à la totalité du milieu de vie auquel ils sont totalement intégrés.

- Les liens avec la forêt ont aussi une transposition symbolique a prendre en compte, qui reflète des cultures bien différentes et montre leur rapport à l'environnement et aux ressources, à la fois au plan matériel et spirituel (Bourguignat et Ribaut, 2000).

- La connaissance assez précise des modes de gestion et activités forestières, qui diffèrent selon les forêts (« écrémage » en forêt tropicale humide, plantations après coupe à blanc, futaie jardinée, futaie régulière, la distinction entre sylviculture et ligniculture), sont des éléments qui permettent de comprendre à la fois les effets environnementaux et la possible adéquation à des stratégies de développement durable.

- Des concepts tels que ceux de «renouvelabilité » et de «durabilité » peuvent ainsi être analysés de façon concrète et pertinente à partir d'exemples relatifs à la forêt, au bois, à l'agroforesterie et à l'extraction.

- En recueillant les représentations des apprenants et en cherchant à les interpréter, on peut aussi mieux analyser les relations culturelles et montrer les évolutions qui ont abouti à l'altération, voire à la disparition de certaines cultures.

\section{Conclusion}

L'éducation relative à l'environnement - bien qu'insuffisamment développée dans nombre de systèmes d'éducation - est un domaine éducatif qui a atteint aujourd'hui une belle maturité, grâce notamment à une réflexion approfondie sur les différentes conceptions qu'on peut en avoir, à une évaluation sérieuse de sa pertinence et de ses impacts et à l'usage de méthodologies appropriées. Le développement personnel de l'individu et sa préparation à l'exercice citoyen sont parmi les préoccupations essentielles de l'ERE: ceci implique une réelle ouverture sur les réalités de notre monde. Or celles-ci, au plan concret et matériel ainsi qu'en ce qui concerne les impacts sur l'environnement, sont d'ordre économique. C'est pourquoi, selon nous, une approche pragmatique de l'ERE doit être privilégiée. Mais aussi, tout en sachant que toute éducation doit se faire avec les plus grandes précautions déontologiques, l'ERE, dont l'une des caractéristiques est la prise en compte des valeurs fondamentales, ne doit pas craindre d'apparaître comme une éducation philosophique de base, utilisant clairement l'analyse critique et le rapport au concret dans une perspective d'éducation relative à l'exercice de la citoyenneté. Ceci implique que l'ERE ne reste pas focalisée sur les jeunes publics en situation scolaire ou parascolaire. Une publication récente par Yolanda Ziaka et coll. (2002) propose de s'intéresser non seulement au système éducatif et à la formation des enseignants et éducateurs, mais encore de chercher à atteindre davantage le grand public par le relais de groupes particuliers (médiatiques, politiques et administratifs, associatifs, entrepreneuriales, professionnels à fort impact sur l'environnement, etc.) afin, en particulier, de créer des « outils collectifs d'acquisition de compétences [pour] tous ceux qui ont un rôle formateur au sens large » (p. 131). 


\section{BIBLIOGRAPHIE}

Bourguignat, E. et Ribaut, J.-P. (2000). L'arbre et la forêt : du symbolisme culturel à... l'agonie programmée + Paris : Éditions Charles Léopold Mayer.

Brodhag, C. (2002). Le développement durable : des concepts à la stratégie française. Dans Collège des Hautes Études de l'Environnement (dir.), Environnement et développement durable : quelles nouvelles compétences pour les dirigeants? Paris : École Centrale de Paris, p. 1-19.

Commission mondiale sur l'environnement et le développement. (1988). Notre avenir à tous. Montréal : Éditions du fleuve.

Deléage, J.-P. et Grinevald, J. (1993). La prise de conscience. Dans M. Beaud, C. Beaud et M.L. Bouguerra (dir.), L'état de l'environnement dans le monde. Paris : La Découverte / FPH, p. 30-43.

Dorst, J. (1965). Avant que nature meure. Neuchâtel : Delachaux et Niestlé.

Erlich, P.R. et Erlich A. (1998). Population, plenty and poverty. Washington D.C. : National Geographic.

Fabre, J.H. (1989). Souvenirs entomologiques : Étude sur l'instinct et les mœurs des insectes. Tome II. Paris : Éditions Robert Laffont.

Goffin, L. (2001). L'éducation relative à l'environnement (ERE) : conception, publics cibles, acteurs et stratégies. Dans F. Debuyst, P. Defourny et H. Gérard (dir.), Savoirs et jeux d'acteurs pour des développements durables. Louvain-la-Neuve et Paris : Académia Bruyland et L'Harmattan, p. 313-339.

Goffin, L. (1999). Érika et l'ERE. Symbioses, 45, 3-7.

Klatzmann, J. (1975). Nourrir dix milliards d'hommes ? Paris : Presses universitaires de France.

Lévi-Strauss, C. (1955). Tristes tropiques. Paris : Plon.

Meadows, D.H. et Delaunay, J. (1972). Halte à la croissance. Paris : A Fayard.

Ramos, C. (2003) La forêt comme thème d'éducation relative à l'environnement : analyse didactique des processus éducatifs. Thèse de doctorat de l'Université de Paris Sud, France.

Sauvé, L. (2002). L'éducation relative à l'environnement : possibilités et contraintes. Connexion, 27 (1-2), 1-3.

Sauvé, L., Orellana, I., Qualman, S. et Dubé, S. (2001). L'éducation relative à l'environnement. École et communauté : une dynamique constructive. Montréal : Hurtubise HMH.

Serres, M. (1990). Le contrat naturel. Paris : Éditions François Bourrin.

Weber, M. (1905). L'éthique protestante et l'esprit du capitalisme. Paris : Plon.

Ziaka, Y., Robichon, P. et Souchon, C. (2001). Éducation à l'environnement : 6 propositions pour agir en citoyens. Série des cahiers de propositions pour le XXIe siècle. Paris : Éditions Fondation Charles Leopold Mayer.

\section{NOTES}

1. Cette proposition éthique se rapproche de celle de Louis Goffin (2001). 
2. Ces éléments théoriques peuvent être associés à la démarche proposée par Louis Goffin (1999).

\section{INDEX}

Keywords : environmental education, political ecology, culture, economy, media, liberalism, sustainable development

Mots-clés : éducation relative à l'environnement, écologie politique, culture, économie, médias, libéralisme, développement durable

\section{AUTEURS}

\section{CARMEN RAMOS}

Carmen Ramos, après avoir été professeur de biologie dans l'enseignement secondaire à Iquitos au Pérou, a obtenu le Diplôme d'études approfondies de didactique de la biologie et de l'éducation à l'environnement de l'Université Paris VII. Elle a ensuite entrepris un travail de recherche appliquée (en liaison avec le groupe EDEN) sur la forêt comme thème d'éducation à l'environnement, présenté pour une thèse de doctorat de l'Université Paris XI soutenue en mars 2003.

\section{CHRISTIAN SOUCHON}

Christian Souchon, après avoir enseigné à l'Université Paris VII jusqu'en 1997, est maintenant directeur-animateur du groupe de recherche et de réflexion EDEN (Éducation pour le Développement et l'ENvironnement) au sein de l'association DIRES (Didactique, innovation, recherche en éducation scientifique). Ses champs de recherche touchent à l'éco- énergétique, aux méthodologies en ERE, à la pédagogie de la modélisation-simulation et à celle des pratiques de l'écotourisme. 